\title{
Non-equilibrium spin-boson model: counting statistics and the heat exchange fluctuation theorem
}

\author{
Lena Nicolin and Dvira Segal \\ Chemical Physics Theory Group, Department of Chemistry, \\ University of Toronto, 80 Saint George St. Toronto, Ontario, Canada M5S $3 H 6$
}

(Dated: October 21, 2018)

\begin{abstract}
We focus on the non-equilibrium two-bath spin-boson model, a toy model for examining quantum thermal transport in many-body open systems. Describing the dynamics within the NIBA equations, applicable, e.g., in the strong system-bath coupling limit and/or at high temperatures, we derive expressions for the cumulant generating function in both the markovian and non-markovian limits by energy-resolving the quantum master equation of the subsystem. For a markovian bath, we readily demonstrate the validity of a steady-state heat exchange fluctuation theorem. In the non-markovian limit a "weaker" symmetry relation generally holds, a general outcome of microreversibility. We discuss the reduction of this symmetry relation to the universal steady-state fluctuation theorem. Using the cumulant generating function, an analytic expression for the heat current is obtained. Our results establish the validity of the steady-state heat exchange fluctuation theorem in quantum systems with strong system-bath interactions. From the practical point of view, this study provides tools for exploring transport characteristics of the two-bath spin-boson model, a prototype for a nonlinear thermal conductor.
\end{abstract}




\section{INTRODUCTION}

Impurity models were proved to be extremely useful in predicting many physical phenomena. The famous spin-boson model $[1,2]$, describing the dynamics a single charge on two states coupled to a dissipative bath, e.g., a solvent, exhibits rich phenomenology, including various phase transitions. Its applications range from charge transfer reactions in biological systems [3], photosynthesis [4], and the Kondo problem for magnetic impurities [5]. A variant of the model is the spin-fermion model, where a qubit (spin) interacts with one or more metallic environments [6-8]. These celebrated impurity models are appealing from various reasons. First, they enclose rich dynamical phenomenology, e.g., the Marcus theory [1] and the Kondo physics [5]. More recently, addressing molecular electronic experiments, such generic models were proved to be useful in predicting various aspects of molecular transport characteristics [9]. Secondly, they serve as a benchmark for developing simulation techniques and approximation schemes, for describing the dynamics of open many-body systems $[10,11]$.

The traditional spin-boson (SB) model, considering an impurity-spin coupled to a single thermal reservoir, serves as a prototype model for exploring quantum dissipation problems [1]. The non-equilibrium version of this model, referring to the case where the spin (subsystem) is coupled to two thermal reservoirs, has been suggested as a toy model for exploring quantum transport phenomenology through an anharmonic nanojunction $[12,13]$. In this case, the generic situation is one of a non-equilibrium steady-state, regardless of the initial preparation. We refer to this model as the "non-equilibrium spin-boson model" (NESB). Given the complex dissipative spin dynamics observed in the single-bath SB model [1], one expect its non-equilibrium extension to reveal tangled transport properties. Fundamental topics of interest are the scaling of the energy current with the spin-bath coupling strength, the role the reservoirs spectral function and the tunneling splitting on the subsystem dynamics and the transport coefficients, and the onset of nonlinear current-temperature bias characteristics at strong interactions.

The transport behavior of the unbiased (zero magnetic field) NESB model has been studied perturbatively, under the assumption of weak system-bath interactions, using master equation methods $[13,14]$. While this scheme, providing simple analytic expressions, can capture some of the aspects of the energy transport process, the inherent weak system- 
bath coupling assumption results in a resonance-sequential transport process where the two reservoirs separately excite and relax the subsystem. Exact numerical results can be obtained by following the Keldysh approach [15] or by using the complex machinery of the multilayer multiconfiguration Hartree approach [16].

In this paper we present an analytical study of the NESB model in the strong coupling limit and/or at high temperatures. In this limit a concerted action of the two baths takes place, where at each relaxation or excitation process both reservoirs contribute in a nonadditive manner. This renders the master equation description complex, since the amount of energy transferred between the two baths is no longer in a one-to-one relationship with the number of spin flip events. The objective of our analysis is the cumulant generating function (CGF). With this at hand, one can derive analytic expressions for the transport coefficients: the current and its cumulants, exposing their dependence on the microscopic parameters. Furthermore, given the CGF, the validity of the steady-state heat exchange fluctuation theorem [17] can be established for anharmonic quantum models in the strong coupling limit.

The fluctuation theorem (FT) for entropy production quantifies the probability of negative entropy generation, measuring "second law violation" [18, 19]. Both transient and steady-state fluctuation theorems (SSFT) have been derived, where the former looks at non steady-state processes over a finite time $t$, and the latter measures entropy production in non-equilibrium steady-state systems over a long interval. In the context of heat exchange between two equilibrium reservoirs, $\nu=L, R$, the SSFT can be roughly stated as $[17,20]$

$$
\ln \left[\mathcal{P}_{t}(+\omega) / \mathcal{P}_{t}(-\omega)\right]=\Delta \beta \omega
$$

Here $\mathcal{P}_{t}(\omega)$ denotes the probability distribution of the net heat transfer $\omega$, from $L$ to $R$, over the (long) interval $t$, with $\Delta \beta=T_{R}^{-1}-T_{L}^{-1}$ as the difference between the inverse temperatures of the reservoirs. Extending the work and heat FT to the quantum domain has recently attracted significant attention [21, 22]. Specifically, a quantum exchange FT, for the transfer of energy between two reservoirs maintained at different temperatures, has been derived in Refs. [17, 23, 24] using projective measurements, and in Refs. [25, 26], based on the unraveling of the quantum master equation (QME). These derivations assume that the interaction between the two thermal baths is weak, and can be neglected with respect to overall energy changes. Using the Keldysh approach, an exact analysis was carried out 
in Ref. [27]. However, it is valid only for harmonic systems. It is thus an open question whether a heat exchange FT is obeyed by an anharmonic quantum system strongly coupled to multiple reservoirs.

Another subtle point is the role of non-markovian effects on the heat exchange SSFT and the current cumulants. In charge transfer problems, this topic has recently attracted significant interest [28-30]. The analogous problem, the reflection of non-markovian effects within the CGF in energy exchange scenarios has been considered for equilibrium systems in Ref. [31]. The Markov approximation is justified once the relaxation of the bath is fast, while the dynamics of the subsystem is slow. In this case the amount of energy transferred between the subsystem and the bath is pinned down with an arbitrary precision, as a strict energy conservation condition is enforced. However, once the assumption of markovianity is relaxed, when the dynamics of the baths degrees of freedom is on a comparable timescale with the subsystem evolution, energy-non-conserving processes on short time scales due to the energy-time uncertainty (when looking only at a subsystem) cannot be excluded. On this bath-decorrelation time scale, it is not obvious that the basic symmetry [Eq. (1)] still holds.

Considering the NESB model in the strong interaction limit, allowing for non-markovian effects, it is our objective here to investigate its heat exchange properties: (i) To obtain the CGF and gain an explicit expression for the heat current, useful for understanding heat current characteristics for anharmonic-strongly coupled systems. (ii) Given the CGF, to derive the heat exchange SSFT. (iii) To understand the role of non-markovian (memory) effects on the onset of the SSFT. Our analysis makes use of the noninteracting-blip approximation (NIBA) [1]. This scheme can faithfully simulate the SB dynamics at strong system-bath interactions and/or at high temperatures in the Ohmic case. It is also exact for the unbiased case at weak damping. Under this approximation, the subsystem's dynamics is described within a time convolution quantum master equation. We unravel this dynamical equations into trajectories with a particular amount of net energy dissipated at each contact. In the markovian limit a heat exchange SSFT is verified. We also obtain the CGF, independent of the particular physical realization. In the non-markovian case a symmetry relation is recovered [22], reaching the universal SSFT once the observation time $t$ [Eq. (1)] is much greater than the bath decorrelation time.

The paper is organized as follows. In Sec. II, we describe our model and recall known 
results for the spin-boson model in the strong coupling limit. Sec. III presents results for the CGF in the markovian limit, introducing the concepts and definitions that will become useful once the more involved non-markovian case is considered in Sec. IV. In Sec. V we conclude.

\section{MODEL AND DYNAMICS}

The non-equilibrium spin-boson Hamiltonian, comprising a spin subsystem coupled to two $(\nu=L, R)$ independent phonon baths, maintained at a temperature $T_{\nu}$, is described by the Hamiltonian $(\hbar \equiv 1)$

$$
H=\frac{\omega_{0}}{2} \sigma_{z}+\frac{\Delta}{2} \sigma_{x}+\sigma_{z} \sum_{\nu, j} \lambda_{j, \nu}\left(b_{j, \nu}^{\dagger}+b_{j, \nu}\right)+\sum_{\nu, j} \omega_{j} b_{j, \nu}^{\dagger} b_{j, \nu} .
$$

Here $\sigma_{x}$ and $\sigma_{z}$ are the Pauli matrices, $\omega_{0}$ is the energy gap between the spin levels, and $\Delta$ is the tunneling energy. Explicitly, in the two-state basis, $\sigma_{z}=|1\rangle\langle 1|-| 0\rangle\langle 0|$ and $\sigma_{x}=|1\rangle\langle 0|+| 0\rangle\langle 1|$. Each reservoir includes a collection of uncoupled harmonic oscillators,

$b_{j, \nu}^{\dagger}\left(b_{j, \nu}\right)$ is the bosonic creation (annihilation) operator of the mode $j$ in the $\nu$ reservoir. The parameter $\lambda_{j, \nu}$ accounts for the system-bath interaction strength.

The transport characteristic of the non-equilibrium spin-boson model can be obtained exactly using numerical simulations [16]. Here, with the motivation to gain insight into the heat current characteristics, the behavior of the current cumulants, and the fluctuation symmetries we resort to approximations, allowing for analytical results. In particular, we employ the NIBA equations, valid at strong system-bath interactions or for high temperatures, assuming an Ohmic spectral density [1,2]. The NIBA equations can be also obtained by applying the Born approximation with respect to the dressed tunneling elements [32, 33]. While this method has been originally derived for a spin coupled to a single bosonic reservoir, one can trivially generalize it to describe a multi-bath case.

We begin by transforming the SB Hamiltonian (2) to the displaced bath-oscillators basis using the small polaron transformation [34], $H_{p}=U^{\dagger} H U, U=e^{i \sigma_{z} \Omega / 2}$,

$$
H_{p}=\frac{\omega_{0}}{2} \sigma_{z}+\frac{\Delta}{2}\left(\sigma_{+} e^{i \Omega}+\sigma_{-} e^{-i \Omega}\right)+\sum_{\nu, j} \omega_{j} b_{j, \nu}^{\dagger} b_{j, \nu},
$$

where $\sigma_{ \pm}=\frac{1}{2}\left(\sigma_{x} \pm i \sigma_{y}\right)$, or $\sigma_{+}=|0\rangle\left\langle 1\left|, \sigma_{-}=\right| 1\right\rangle\langle 0|$, are the auxiliary Pauli matrices, $\Omega=\sum_{\nu} \Omega_{\nu}$, and $\Omega_{\nu}=2 i \sum_{j} \frac{\lambda_{j, \nu}}{\omega_{j}}\left(b_{j, \nu}^{\dagger}-b_{j, \nu}\right)$. Under the NIBA approximation $[2,32,33]$, 
generalized to the two-baths case, it can be shown that the spin polarization $\left\langle\sigma_{z}(t)\right\rangle$ obeys a convolution-type master equation

$$
\frac{d\left\langle\sigma_{z}\right\rangle}{d t}=-\int_{0}^{t} K_{s}(t-\tau)\left\langle\sigma_{z}(\tau)\right\rangle d \tau-\int_{0}^{t} K_{a}(t-\tau) d \tau
$$

where the symmetric and antisymmetric kernels are given by

$$
\begin{aligned}
& K_{s}(t)=\Delta^{2} e^{-Q^{\prime}(t)} \cos \left[Q^{\prime \prime}(t)\right] \cos \left(\omega_{0} t\right) \\
& K_{a}(t)=\Delta^{2} e^{-Q^{\prime}(t)} \sin \left[Q^{\prime \prime}(t)\right] \sin \left(\omega_{0} t\right)
\end{aligned}
$$

The complex function $Q(t)=\sum_{\nu} Q_{\nu}(t)$, made of a real and imaginary components, $Q_{\nu}(t)=$ $Q_{\nu}^{\prime}(t)+i Q_{\nu}^{\prime \prime}(t)$, is defined by the correlation function $e^{-Q(t)}=\left\langle e^{i \Omega(t)} e^{-i \Omega(0)}\right\rangle$, with the thermal average performed over both reservoirs degrees of freedom. It is given by

$$
\begin{aligned}
& Q_{\nu}^{\prime \prime}(t)=\int_{0}^{\infty} \frac{J_{\nu}(\omega)}{\pi \omega^{2}} \sin (\omega t) d \omega \\
& Q_{\nu}^{\prime}(t)=\int_{0}^{\infty} \frac{J_{\nu}(\omega)}{\pi \omega^{2}}[1-\cos (\omega t)]\left[1+2 n_{\nu}(\omega)\right] d \omega
\end{aligned}
$$

Here $J_{\nu}(\omega)$ is the $\nu$-bath spectral function, incorporating system-bath interactions

$$
J_{\nu}(\omega)=4 \pi \sum_{j} \lambda_{j, \nu}^{2} \delta\left(\omega-\omega_{j}\right)
$$

In what follows we focus on the two-state population dynamics, therefore we rewrite Eq. (4) in terms of the states population

$$
\begin{aligned}
\frac{d p_{1}(t)}{d t} & =-\frac{\Delta^{2}}{2} \int_{0}^{t} e^{-Q^{\prime}(t-s)} \cos \left[\omega_{0}(t-s)-Q^{\prime \prime}(t-s)\right] p_{1}(s) d s \\
& +\frac{\Delta^{2}}{2} \int_{0}^{t} e^{-Q^{\prime}(t-s)} \cos \left[\omega_{0}(t-s)+Q^{\prime \prime}(t-s)\right] p_{0}(s) d s \\
1 & =p_{0}(t)+p_{1}(t),
\end{aligned}
$$

where $\left\langle\sigma_{z}(t)\right\rangle=p_{1}(t)-p_{0}(t)$. We explore next the heat transport characteristics under the NIBA approximation (i) assuming a markovian dynamics, and (ii) more generally, retracting to the non-markovian case, allowing for memory effects in the thermal baths. The nonmarkovian analysis can be reduced to the markovian description in the appropriate limit. For clarity, we have decided to first present here the (simple) markovian limit, then generalize the analysis and portray the non-markovian regime. This allows us to introduce the main concepts involved in the CGF derivation within a relatively simple setup. 


\section{MARKOVIAN LIMIT}

\section{A. Population Dynamics}

A general analysis of counting statistics of a multi-state system connecting two nonequilibrium markovian reservoirs has been carried out in Ref. [35], based upon the NIBA equations. We use this scheme and derive here the CGF for the NESB model. In the markovian limit one assumes that the spin system slowly evolves in comparison to the reservoirs evolution. Thus, we make the following two simplifications in the integro-differential equation (8): First, we replace the population, $p_{n}(s)$ by $p_{n}(t)(n=0,1)$, supposing that the timescale over which the memory, represented by the integral, is important, is suffi-

ciently short. Second, we extend the integral upper limit to infinity, assuming the integrand quickly dies out. Under these approximations, Eq. (8) reduces to a kinetic equation for the population dynamics,

$$
\dot{p}_{1}=-k_{d} p_{1}(t)+k_{u} p_{0}(t)
$$

The rate constants are given as Fourier transforms of bath correlation functions,

$$
k_{d}=C\left(\omega_{0}\right), \quad k_{u}=C\left(-\omega_{0}\right)
$$

with

$$
C\left(\omega_{0}\right)=\int_{-\infty}^{\infty} e^{i \omega_{0} t} C_{L}(t) C_{R}(t) d t .
$$

The ingredients of this correlation function are given in terms of the function $Q_{\nu}(t)$, defined in Eq. (6),

$$
C_{\nu}(t)=\frac{\Delta}{2} e^{-Q_{\nu}(t)}
$$

Using the convolution theorem, the transition rates $C\left( \pm \omega_{0}\right)$ can be rewritten as a convolution of the $L$-bath and $R$-bath induced processes,

$$
C\left(\omega_{0}\right)=\frac{1}{2 \pi} \int_{-\infty}^{\infty} C_{L}\left(\omega_{0}-\omega\right) C_{R}(\omega) d \omega
$$

introducing the Fourier transform

$$
C_{\nu}(\omega)=\int_{-\infty}^{\infty} e^{i \omega t} C_{\nu}(t) d t
$$


These bath-specific microscopic rates satisfy the detailed balance relation,

$$
\frac{C_{\nu}(\omega)}{C_{\nu}(-\omega)}=e^{\omega \beta_{\nu}}
$$

However, such a relation does not hold for the combined rate $C(\omega)$, ruling the dynamics. The QME (9) encloses complex physical processes as Eq. (13) draws nontrivial transfer rates. When the system decays it disposes the energy $\omega_{0}$ into both reservoirs, cooperatively; the energy $\omega$ is dissipated into the $R$ bath while the $L$ bath gains (or contributes) the rest, $\omega_{0}-\omega$. Similarly, excitation of the system occurs through an $L-R$ compound process. Since energy is dissipated or absorbed in such complex processes, energy "counting" is a nontrivial task, as reflected in the resolved master equation (16) discussed below.

\section{B. Cumulant Generating Function}

We construct next the cumulant generating function for the NESB model in the NIBAmarkovian limit presented above. Following Ref. [26], we begin by defining the function $\mathcal{P}_{t}(n, \omega)$ as the probability that within the time $t$ a total of energy $\omega$ has been transferred from the left bath to the right bath, while the spin is populating the $n(n=0,1)$ state at time $t$. The time evolution of this quantity follows

$$
\begin{aligned}
\frac{d \mathcal{P}_{t}(0, \omega)}{d t} & =-\mathcal{P}_{t}(0, \omega) \int_{-\infty}^{\infty} \frac{1}{2 \pi} C_{R}(-\tilde{\omega}) C_{L}\left(\tilde{\omega}-\omega_{0}\right) d \tilde{\omega} \\
& +\int_{-\infty}^{\infty} \frac{1}{2 \pi} C_{R}(\omega-\tilde{\omega}) C_{L}\left(\omega_{0}-(\omega-\tilde{\omega})\right) \mathcal{P}_{t}(1, \tilde{\omega}) d \tilde{\omega} \\
\frac{d \mathcal{P}_{t}(1, \omega)}{d t} & =-\mathcal{P}_{t}(1, \omega) \int_{-\infty}^{\infty} \frac{1}{2 \pi} C_{R}(\omega) C_{L}\left(\omega_{0}-\omega\right) d \omega \\
& +\int_{-\infty}^{\infty} \frac{1}{2 \pi} C_{R}(\omega-\tilde{\omega}) C_{L}\left((\tilde{\omega}-\omega)-\omega_{0}\right) \mathcal{P}_{t}(0, \tilde{\omega}) d \tilde{\omega}
\end{aligned}
$$

for details see Appendix A. One can rationalize this equation as follows. Focusing for example on the dynamics of $\mathcal{P}_{t}(1, \omega)$, the first term in this rate equation describes the decay of this probability as the spin flips to the ground state and extra energy is dissipated to the $R$ reservoir. The second term collects processes with an energy $\tilde{\omega}$ transferred to the $R$ bath by the time $t$, with the spin occupying the ground state. At time $t$ a spin flip takes place accompanied by an extra energy $\omega-\tilde{\omega}$ dissipated to the $R$ bath, completing the transfer of a total amount of energy $\omega$ to the right bath at $t$. 
We note that in the present model we cannot adopt standard approaches for unraveling the reduced density matrix, directly dressing the interaction term in the Hamiltonian by the counting process [21]. This in because the two reservoirs affect the energy transfer process in a nonlinear way, thus counting system-bath interaction processes (as in a perturbation theory series) does not reveal here the actual amount of energy exchanged between the two reservoirs.

We Fourier transform the above system of equations to obtain the characteristic function $Z(\chi, t)$ for the energy counting field $\chi$,

$$
|Z(\chi, t)\rangle \equiv\left(\begin{array}{l}
\int_{-\infty}^{\infty} \mathcal{P}_{t}(0, \omega) e^{i \omega \chi} d \omega \\
\int_{-\infty}^{\infty} \mathcal{P}_{t}(1, \omega) e^{i \omega \chi} d \omega
\end{array}\right)
$$

It satisfies the differential equation

$$
\frac{d|Z(\chi, t)\rangle}{d t}=-\hat{W}(\chi)|Z(\chi, t)\rangle
$$

where the matrix $\hat{W}$ contains the following elements

$$
\hat{W}(\chi)=\left(\begin{array}{cc}
C\left(-\omega_{0}\right) & -C^{d}(\chi) \\
-C^{u}(\chi) & C\left(\omega_{0}\right)
\end{array}\right)
$$

The diagonal terms were defined above, see Eq. (11). The nondiagonal terms are given by the integrals

$$
C^{d / u}(\chi)=\frac{1}{2 \pi} \int_{-\infty}^{\infty} C^{d / u}(\omega) e^{i \omega \chi} d \omega
$$

with the components

$$
\begin{aligned}
& C^{d}(\omega)=C_{R}(\omega) C_{L}\left(\omega_{0}-\omega\right) \\
& C^{u}(\omega)=C_{R}(\omega) C_{L}\left(-\omega-\omega_{0}\right) .
\end{aligned}
$$

The cumulant generating function is formally defined as

$$
G(\chi)=\lim _{t \rightarrow \infty} \frac{1}{t} \ln \int_{-\infty}^{\infty} \mathcal{P}_{t}(\omega) e^{i \omega \chi} d \omega
$$

where we introduced the short notation, $\mathcal{P}_{t}(\omega)=P_{t}(0, \omega)+P_{t}(1, \omega)$, the probability to transfer by the time $t$ an energy $\omega$ from left to right, irrespective of the spin state. In the present case the CGF is expressed in terms of $|Z\rangle$ as

$$
G(\chi)=\lim _{t \rightarrow \infty} \frac{1}{t} \ln \langle I \mid Z(\chi, t)\rangle
$$


with $\langle I|=\langle 11|$, denoting a left vector of unity. It is practically given by the negative of the smallest eigenvalue of the matrix $\hat{W}$. We diagonalize $\hat{W}$ and explicitly obtain the CGF in terms of the microscopic rates,

$$
G(\chi)=-\frac{C\left(\omega_{0}\right)+C\left(-\omega_{0}\right)}{2}+\frac{\left[\left(C\left(\omega_{0}\right)-C\left(-\omega_{0}\right)\right)^{2}+4 C^{d}(\chi) C^{u}(\chi)\right]^{1 / 2}}{2} .
$$

The heat current and its noise power can be readily derived, by taking the first and the second derivatives, respectively, of the CGF

$$
\begin{aligned}
& \langle J\rangle \equiv \frac{\langle\omega\rangle_{t}}{t}=\left.\frac{d G(\chi)}{d(i \chi)}\right|_{\chi=0}, \\
& \langle S\rangle \equiv \frac{\left\langle\omega^{2}\right\rangle_{t}-\langle\omega\rangle_{t}^{2}}{t}=\left.\frac{d^{2} G(\chi)}{d(i \chi)^{2}}\right|_{\chi=0} .
\end{aligned}
$$

Here $\langle\omega\rangle_{t}$ denotes the total energy $\omega$ transferred from $L$ to $R$ by the (infinitely long) time t. Using the formal structure (24) one can show that the steady-state heat current, defined as positive when flowing left to right, obeys

$$
\langle J\rangle=\frac{1}{2 \pi} \int_{-\infty}^{\infty} \omega d \omega\left[C_{R}(\omega) C_{L}\left(\omega_{0}-\omega\right) p_{1}-C_{R}(-\omega) C_{L}\left(-\omega_{0}+\omega\right) p_{0}\right] .
$$

This expression incorporates the steady-state populations

$$
p_{1}=C\left(-\omega_{0}\right) /\left(C\left(\omega_{0}\right)+C\left(-\omega_{0}\right)\right), \quad p_{0}=C\left(\omega_{0}\right) /\left(C\left(\omega_{0}\right)+C\left(-\omega_{0}\right)\right) .
$$

For details see Appendix B. The result for the heat current agrees with the expression used ad-hoc in Refs. $[12,13]$. It can be rationalized by viewing $\omega C_{R}(\omega) C_{L}\left(\omega_{0}-\omega\right) p_{1}$ as a spin relaxation process with the energy $\omega$ directed to the $R$ bath and the amount of $\omega_{0}-\omega$ disposed into the $L$ bath. Similarly, the second term describes energy loss from the $R$ bath, where, combined with an energy influx from the $L$ bath, results in the excitation of the spin system. It is significant to note that this expression has been achieved under relatively general conditions, for systems satisfying a markovian-NIBA approximation. The details of the Kernel $K_{s / a}(t)$ (e.g., the bath statistics) are not utilized in this derivation. Thus, it is valid for other systems following the structure (9)-(11), e.g., the spin-fermion model $[6,7,35]$. Appendix B further details the derivation of the the second cumulant, the noise 
power of the NESB junction,

$$
\begin{aligned}
\langle S\rangle & =p_{1} \int_{-\infty}^{\infty} \frac{1}{2 \pi} \omega^{2} C_{R}(\omega) C_{L}\left(\omega_{0}-\omega\right) d \omega+p_{0} \int_{-\infty}^{\infty} \frac{1}{2 \pi} \omega^{2} C_{R}(-\omega) C_{L}\left(\omega-\omega_{0}\right) d \omega+ \\
& -2 \frac{1}{C\left(\omega_{0}\right)+C\left(-\omega_{0}\right)} \frac{1}{(2 \pi)^{2}} \int_{-\infty}^{\infty} \omega C_{R}(-\omega) C_{L}\left(\omega-\omega_{0}\right) d \omega \int_{-\infty}^{\infty} \omega C_{R}(\omega) C_{L}\left(\omega_{0}-\omega\right) d \omega+ \\
& -2 \frac{1}{C\left(\omega_{0}\right)+C\left(-\omega_{0}\right)}\langle J\rangle^{2} .
\end{aligned}
$$

\section{Fluctuation Theorem}

We continue and confirm the validity of the SSFT in the NESB model, under the Markov approximation. This relation can be established by examining the symmetry of the CGF, Eq. (24) [21]. It is clear that it is sufficient to focus on the product term, $\mathcal{D}(\chi) \equiv C^{d}(\chi) C^{u}(\chi)$, for

resolving the symmetry of $G(\chi)$. Using the definitions (20)-(21) we therefore write (ignoring the $2 \pi$ prefactors)

$$
\mathcal{D}(\chi)=\int_{-\infty}^{\infty} e^{i \omega \chi} C_{R}(\omega) C_{L}\left(\omega_{0}-\omega\right) d \omega \times \int_{-\infty}^{\infty} e^{i \omega \chi} C_{R}(\omega) C_{L}\left(-\omega_{0}-\omega\right) d \omega .
$$

Shifting the argument $\chi \rightarrow(i \Delta \beta-\chi), \Delta \beta=\beta_{R}-\beta_{L}$, it translates to

$$
\begin{aligned}
\mathcal{D}(i \Delta \beta-\chi) & =\int_{-\infty}^{\infty} e^{-i \omega \chi} e^{-\omega \Delta \beta} C_{R}(\omega) C_{L}\left(\omega_{0}-\omega\right) d \omega \\
& \times \int_{-\infty}^{\infty} e^{-i \omega \chi} e^{-\omega \Delta \beta} C_{R}(\omega) C_{L}\left(-\omega_{0}-\omega\right) d \omega .
\end{aligned}
$$

We now change variables, $\omega \rightarrow-\omega$, then use the detailed balance relation for $C_{\nu}(\omega)$, see Eq. (15). This transforms the first element in the RHS of the above equation to

$$
\begin{aligned}
& C^{d}(i \Delta \beta-\chi)=\int_{-\infty}^{\infty} e^{i \omega \chi} e^{\omega \Delta \beta} C_{R}(-\omega) C_{L}\left(\omega_{0}+\omega\right) d \omega \\
& =\int_{-\infty}^{\infty} e^{i \omega \chi} e^{\omega \Delta \beta} C_{R}(\omega) e^{-\beta_{R} \omega} C_{L}\left(-\omega_{0}-\omega\right) e^{\beta_{L}\left(\omega_{0}+\omega\right)} d \omega \\
& =e^{\beta_{L} \omega_{0}} C^{u}(\chi) .
\end{aligned}
$$

Similarly, the second element in the RHS of Eq. (30) reduces to

$$
\begin{aligned}
& C^{u}(i \Delta \beta-\chi)=\int_{-\infty}^{\infty} e^{i \omega \chi} e^{\omega \Delta \beta} C_{R}(-\omega) C_{L}\left(\omega-\omega_{0}\right) d \omega \\
& =\int_{-\infty}^{\infty} e^{i \omega \chi} e^{\omega \Delta \beta} C_{R}(\omega) e^{-\beta_{R} \omega} C_{L}\left(\omega_{0}-\omega\right) e^{-\beta_{L}\left(\omega_{0}-\omega\right)} d \omega \\
& =e^{-\beta_{L} \omega_{0}} C^{d}(\chi) .
\end{aligned}
$$


Joining these two pieces we conclude that

$$
C^{d}(\chi) C^{u}(\chi)=C^{d}(i \Delta \beta-\chi) C^{u}(i \Delta \beta-\chi) .
$$

Therefore, the CGF overall satisfies

$$
G(\chi)=G(i \Delta \beta-\chi) .
$$

We are now in position to demonstrate the validity of a fluctuation relation for this nonequilibrium strongly coupled system. The probability to transfer the energy $\omega$ by the time $t$, from $L$ to $R$ is given by the inverse Fourier transform of Eq. (22),

$$
\mathcal{P}_{t}(\omega)=\frac{1}{2 \pi} \int_{-\infty}^{\infty} e^{t G(\chi)} e^{-i \omega \chi} d \chi .
$$

Similarly, the quantity $\mathcal{P}_{t}(-\omega)$ represents the probability that overall an energy $\omega$ has been transmitted in the opposite direction, right to left, up to time $t$. Based on the symmetry of the CGF, Eq. (33), one readily concludes that

$$
\lim _{t \rightarrow \infty} \frac{1}{t} \ln \frac{\mathcal{P}_{t}(\omega)}{\mathcal{P}_{t}(-\omega)}=\omega \Delta \beta .
$$

This expression describes a fluctuation relation for the non-equilibrium SB model, valid beyond the weak-coupling approximation $[25,26]$. Comparing this result to the weak coupling limit, described in Appendix C, we observe that formally these two expressions are identical. However, one should note that in the strong coupling limit the energy variable $\omega$ is continuous, since multi-phonon processes in which part of the energy goes to $L$ bath and part goes to the $R$ baths, are allowed. In contrast, in the weak coupling limit energy transfer processes take place in integer units of the spin spacing, since this energy travels to either reservoirs separately.

\section{Examples and the Gaussian-Marcus limit}

We exemplify our results, and work out an expression for the heat current and the noise power for a specific case, the so called "Marcus" limit [3], assuming high temperatures $T_{\nu}>\omega_{0}$ and strong coupling. This limit is reached by performing a short time expansion of $Q(t),[$ Eq. (6)] resulting in

$$
Q_{\nu}^{\prime}(t)=E_{r}^{\nu} T_{\nu} t^{2}, \quad Q_{\nu}^{\prime \prime}(t)=E_{r}^{\nu} t .
$$


The reorganization energy $E_{r}^{\nu}=\sum_{j} 4 \lambda_{j, \nu}^{2} / \omega_{j}$ incorporates system-bath interactions. It can be equivalently expressed in terms of the spectral density (7), $E_{r}^{\nu}=\int \frac{J_{\nu}(\omega)}{\pi \omega} d \omega$. Using these expressions, the Fourier transform of the time dependent rates (11) and (12) can be resolved,

$$
\begin{aligned}
C_{\nu}(\omega) & =\frac{\Delta}{2} \sqrt{\frac{\pi}{E_{r}^{\nu} T_{\nu}}} \exp \left[-\frac{\left(\omega-E_{r}^{\nu}\right)^{2}}{4 T_{\nu} E_{r}^{\nu}}\right], \\
C\left(\omega_{0}\right) & =\frac{\Delta^{2}}{4} \sqrt{\frac{\pi}{E_{r}^{R} T_{R}+E_{r}^{L} T_{L}}} \exp \left[-\frac{\left(\omega_{0}-E_{r}^{L}-E_{r}^{R}\right)^{2}}{4\left(T_{R} E_{r}^{R}+T_{L} E_{r}^{L}\right)}\right] .
\end{aligned}
$$

Following Eq. (26), the average heat current can be analytically obtained

$$
\langle J\rangle=\Delta^{2} \frac{\sqrt{2 \pi} E_{r}^{L} E_{r}^{R} \Delta T}{\left(2 E_{r}^{L} T_{L}+2 E_{r}^{R} T_{R}\right)^{\frac{3}{2}}} \exp \left[-\frac{\left(E_{r}^{L}+E_{r}^{R}-\omega_{0}\right)^{2}}{4\left(E_{r}^{L} T_{L}+E_{r}^{R} T_{R}\right)}\right] \times f_{A}
$$

where $f_{A}=\left\{\exp \left[\frac{\omega_{0}\left(E_{r}^{L}+E_{r}^{R}\right)}{\left(E_{r}^{L} T_{L}+E_{r}^{R} T_{R}\right)}\right]+1\right\}^{-1}$. This result agrees with $[12,13]$. The second moment of the current can be similarly calculated, however the expression is too cumbersome to be included. We present the behavior of the current and its noise power in Fig. 1. We observe nonlinear effects in the energy current, including the effect of negative differential conductance $[12,13]$. The noise drops with increasing bias temperature.

One can in principle seek to derive an analytic form for the probability distribution function $\mathcal{P}_{t}(\omega)$, since the analytic structure of $\hat{W}$ is known: The diagonal terms are the rates, (38), the nondiagonal part is given by

$$
C^{d / u}(\chi)=\frac{\Delta^{2}}{4} \sqrt{\frac{\pi}{E_{L} T_{L}+E_{R} T_{R}}} e^{-\frac{E_{L}^{2}+E_{R}^{2}+\omega_{0}^{2}+2 E_{L}\left[E_{R}\left(1-2 i \chi \Delta T+2 \chi^{2} T_{L} T_{R}\right) \mp \omega_{0}\right]-2 E_{R} \omega_{0} \mp 4 i E_{R} \chi T_{R} \omega_{0}}{4\left(E_{L} T_{L}+E_{R} T_{R}\right)}}
$$

Since the result is very complex, we retreat here to numerical simulations. We plug these expressions into the formal solution (24) and perform a numerical inverse Fourier transform [Eq. (35)], to obtain the distribution $\mathcal{P}_{t}(\omega)$. Fig. 2 demonstrates its shape at different times. The averaged current and noise agree with the values provided in Fig. 1. We have also confirmed that the different curves indeed satisfy the SSFT (inset).

We now go beyond the Marcus limit, and demonstrate the behavior of the current with the reorganization energy $E_{r}$, quantifying system-bath coupling strength. We consider the unbiased case $\omega_{0}=0$, and take an Ohmic spectral function $J_{\nu}(\omega)=\frac{\pi E_{r}^{\nu}}{\omega_{c}} \omega e^{-\omega / \omega_{c}}$, identical for the two baths. Since we are interested in the energy current behavior for both weak and strong coupling strengths, we numerically calculate the elements in Eq. (26) using the definitions (6) and (14). The results are displayed in Fig. 3 showing a turnover behavior, where the current decays with increasing $E_{r}$, at large values. It can be easily proved that 
the weak coupling scheme can only produce a linear dependence of the current on the coupling strength $[12,13]$, see also Eq. (C18). The decaying behavior observed here is thus a fingerprint of the strong coupling limit. Similar results were reported in [16], using exact numerical simulations. Practically, this turnover behavior indicates that for maximizing the rate of energy transport in nanodevices one should work at the intermediate system-bath coupling limit.
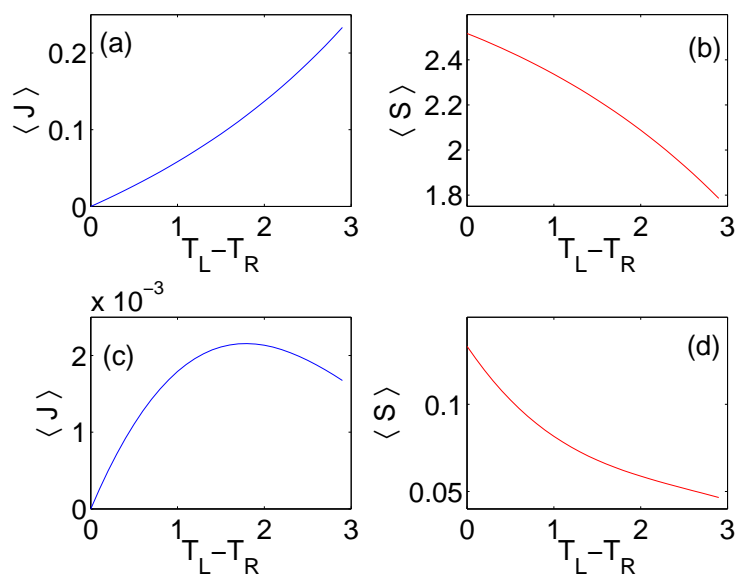

FIG. 1: Energy current and noise power of the spin-boson model in the Marcus limit, Eqs. (38)(40). Other parameters are $T_{L}=5, T_{R}=T_{L}-\Delta T, \omega_{0}=0.5, \Delta / 2=1$, (a)-(b) $E_{r}^{\nu}=1$, (c)-(d) $E_{r}^{\nu}=50$.

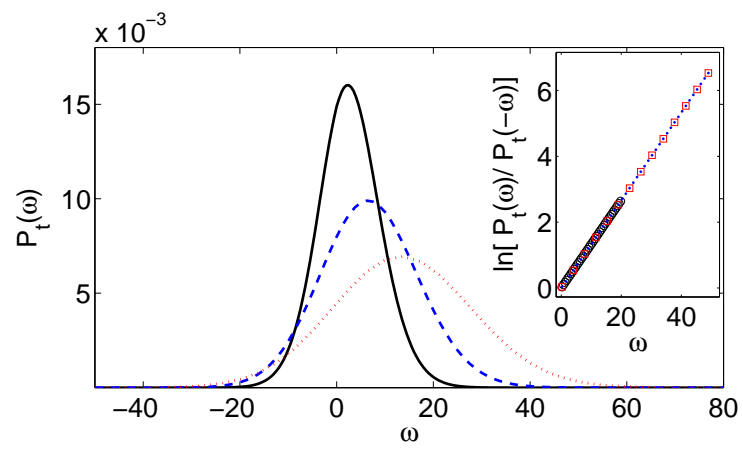

FIG. 2: The probability distribution $\mathcal{P}_{\tau}(\omega)$ at various times, $t=20$ (full), $t=50$ (dashed), $t=100$ (dotted). Other parameters are $T_{L}=5, T_{R}=3, E_{r}^{\nu}=1, \omega_{0}=0.5, \Delta / 2=1$. Data was generated using the Marcus rates. The inset demonstrates for the same data the validity of the fluctuation theorem, with the slope of $\Delta \beta=0.133, t=20$ (o), $t=50$ (dotted), $t=100$ ( $\square$ ). 


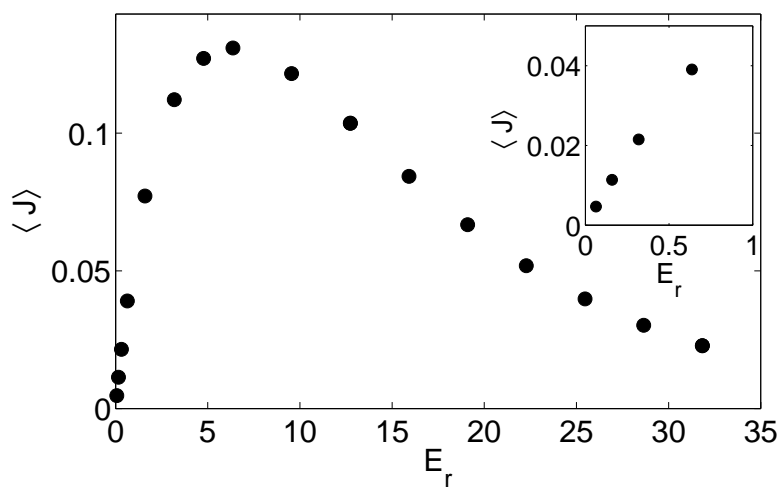

FIG. 3: Energy current in the unbiased spin-boson model, $T_{L}=5, T_{R}=3, \omega_{0}=0, \omega_{c}=10$, $\Delta / 2=1$, numerically simulating (6) and the resulting current (26). The inset zooms on the weak coupling limit, displaying a linear dependency of the heat current on the reorganization energy.

\section{NON-MARKOVIAN DYNAMICS}

\section{A. Cumulant Generating Function}

We generalize here the results of the markovian analysis and derive the CGF for the non-markovian model introduced in Sec. II. A systematic formalism for analyzing nonmarkovian effects in charge transfer systems has been detailed in Refs. [28-30]. Here we adapt this scheme to describe energy transfer processes. Further, while a single counting field has been introduced in [28], in the present model one should introduce two such fields, independently counting energy transmission at each contact. We begin the analysis by rewriting the equation of motion for the spin population (8) as

$$
\begin{aligned}
\frac{d p_{1}(t)}{d t} & =-\frac{\Delta^{2}}{2} \Re \int_{0}^{t} e^{i \omega_{0}(t-s)} e^{-Q_{L}(t-s)} e^{-Q_{R}(t-s)} p_{1}(s) d s \\
& +\frac{\Delta^{2}}{2} \Re \int_{0}^{t} e^{i \omega_{0}(t-s)} e^{-Q_{L}(s-t)} e^{-Q_{R}(s-t)} p_{0}(s) d s, \\
1 & =p_{0}(t)+p_{1}(t),
\end{aligned}
$$

where we made use of the symmetry properties of the $Q(t)$ function, $Q^{\prime}(t-s)=Q^{\prime}(s-t)$ and $Q^{\prime \prime}(t-s)=-Q^{\prime \prime}(s-t)$, see the explicit expressions in Eq. (6). Here $\Re$ denotes the real part. In the next step, we use the Fourier transform relation

$$
\frac{\Delta}{2} e^{-Q_{\nu}(t)}=\frac{1}{2 \pi} \int_{-\infty}^{\infty} e^{-i \omega t} C_{\nu}(\omega) d \omega
$$


and write

$$
\begin{aligned}
\frac{d p_{1}(t)}{d t} & =-\frac{1}{2 \pi^{2}} \Re \int_{0}^{t} d s p_{1}(s) e^{i \omega_{0}(t-s)} \int_{-\infty}^{\infty} d \omega_{1} C_{L}\left(\omega_{1}\right) e^{-i \omega_{1}(t-s)} \int_{-\infty}^{\infty} d \omega_{2} C_{R}\left(\omega_{2}\right) e^{-i \omega_{2}(t-s)} \\
& +\frac{1}{2 \pi^{2}} \Re \int_{0}^{t} d s p_{0}(s) e^{-i \omega_{0}(s-t)} \int_{-\infty}^{\infty} d \omega_{1} C_{L}\left(\omega_{1}\right) e^{-i \omega_{1}(s-t)} \int_{-\infty}^{\infty} d \omega_{2} C_{R}\left(\omega_{2}\right) e^{-i \omega_{2}(s-t)} .
\end{aligned}
$$

We now energy-resolve this equation, $p_{n}(t)=\int_{-\infty}^{\infty} d \omega_{L} \int_{-\infty}^{\infty} d \omega_{R} \mathcal{P}_{t}\left(n, \omega_{L}, \omega_{R}\right)$, looking for the probability $\mathcal{P}_{t}\left(n, \omega_{L}, \omega_{R}\right)$ at the time $t$ the spin occupies the $n(n=0,1)$ state, an overall energy $\omega_{R}$ has been transferred to the $R$ bath and $\omega_{L}$ has been transferred to the left bath. Note that unlike the Markov case, we separately count the energy dissipated at each bath. This probability satisfies the differential equation

$$
\begin{aligned}
& \frac{d \mathcal{P}_{t}\left(1, \omega_{L}, \omega_{R}\right)}{d t}=-\frac{1}{2 \pi^{2}} \int_{0}^{t} d s \mathcal{P}_{s}\left(1, \omega_{L}, \omega_{R}\right) \int_{-\infty}^{\infty} \int_{-\infty}^{\infty} d \omega_{1} d \omega_{2} C_{L}\left(\omega_{1}\right) C_{R}\left(\omega_{2}\right) \Re\left[e^{i\left(\omega_{0}-\omega_{1}-\omega_{2}\right)(t-s)}\right] \\
& +\frac{1}{2 \pi^{2}} \int_{0}^{t} d s \int_{-\infty}^{\infty} \int_{-\infty}^{\infty} d \omega_{1} d \omega_{2} \mathcal{P}_{s}\left(0, \omega_{1}, \omega_{2}\right) C_{R}\left(\omega_{R}-\omega_{2}\right) C_{L}\left(\omega_{L}-\omega_{1}\right) \Re\left[e^{i\left(\omega_{0}+\omega_{R}-\omega_{2}+\omega_{L}-\omega_{1}\right)(t-s)}\right],
\end{aligned}
$$

where we used the fact that $C_{\nu}(\omega)$ is a real function. An analogous equation can be written for the time evolution of the probability $\mathcal{P}_{t}\left(0, \omega_{L}, \omega_{R}\right)$. We now introduce two counting fields $\chi_{L}$ and $\chi_{R}$, for each reservoir, and Fourier transform the above equation with respect to these two fields. Further, we Laplace transform the resulting equation, $H(z)=\int_{0}^{\infty} e^{-z t} h(t) d t$. Utilizing Fourier transform and Laplace transform convolution relations, Eq. (44) reduces to a linear equation

$$
\left|Z\left(\chi_{L}, \chi_{R}, z\right)\right\rangle=\frac{1}{z-\hat{W}\left(\chi_{L}, \chi_{R}, z\right)}\left(z \mid Z\left(\chi_{L}, \chi_{R}, z\right\rangle\right)_{z \rightarrow \infty}
$$

with the initial value theorem invoked, $h(t=0)=\lim _{z \rightarrow \infty} z H(z)$. The vector $|Z\rangle$ is defined by

$$
\left|Z\left(\chi_{L}, \chi_{R}, z\right)\right\rangle \equiv\left(\begin{array}{l}
\int_{0}^{\infty} d t e^{-z t} \int_{-\infty}^{\infty} \mathcal{P}_{t}\left(0, \omega_{L}, \omega_{R}\right) e^{i \omega_{L} \chi_{L}} e^{i \omega_{R} \chi_{R}} d \omega_{L} d \omega_{R} \\
\int_{0}^{\infty} d t e^{-z t} \int_{-\infty}^{\infty} \mathcal{P}_{t}\left(1, \omega_{L}, \omega_{R}\right) e^{i \omega_{L} \chi_{L}} e^{i \omega_{R} \chi_{R}} d \omega_{L} d \omega_{R}
\end{array}\right)
$$

and the kernel $\hat{W}$ represents the matrix

$$
\hat{W}\left(\chi_{L}, \chi_{R}, z\right)=\left(\begin{array}{cc}
-\gamma^{+}(z) & \alpha^{-}\left(\chi_{L}, \chi_{R}, z\right) \\
\alpha^{+}\left(\chi_{L}, \chi_{R}, z\right) & -\gamma^{-}(z)
\end{array}\right)
$$


with the elements

$$
\begin{aligned}
\alpha^{+}\left(\chi_{L}, \chi_{R}, z\right) & =\frac{1}{2 \pi^{2}} \int_{-\infty}^{\infty} \int_{-\infty}^{\infty} e^{i \omega_{1} \chi_{L}} e^{i \omega_{2} \chi_{R}} C_{R}\left(\omega_{2}\right) C_{L}\left(\omega_{1}\right) \frac{z}{z^{2}+\left(\omega_{0}+\omega_{1}+\omega_{2}\right)^{2}} d \omega_{1} d \omega_{2} \\
\alpha^{-}\left(\chi_{L}, \chi_{R}, z\right) & =\frac{1}{2 \pi^{2}} \int_{-\infty}^{\infty} \int_{-\infty}^{\infty} e^{i \omega_{1} \chi_{L}} e^{i \omega_{2} \chi_{R}} C_{R}\left(\omega_{2}\right) C_{L}\left(\omega_{1}\right) \frac{z}{z^{2}+\left(\omega_{0}-\omega_{1}-\omega_{2}\right)^{2}} d \omega_{2} \\
\gamma^{+}(z) & =\frac{1}{2 \pi^{2}} \int_{-\infty}^{\infty} \int_{-\infty}^{\infty} C_{R}\left(\omega_{2}\right) C_{L}\left(\omega_{1}\right) \frac{z}{z^{2}+\left(\omega_{0}+\omega_{1}+\omega_{2}\right)^{2}} d \omega_{1} d \omega_{2} \\
\gamma^{-}(z) & =\frac{1}{2 \pi^{2}} \int_{-\infty}^{\infty} \int_{-\infty}^{\infty} C_{R}\left(\omega_{2}\right) C_{L}\left(\omega_{1}\right) \frac{z}{z^{2}+\left(\omega_{0}-\omega_{1}-\omega_{2}\right)^{2}} d \omega_{1} d \omega_{2}
\end{aligned}
$$

We are interested in the total probability, to occupy either states,

$$
\mathcal{P}_{t}\left(\omega_{L}, \omega_{R}\right) \equiv \sum_{n=0,1} P_{t}\left(n, \omega_{L}, \omega_{R}\right)
$$

We express it in terms of the characteristic function $e^{S\left(\chi_{L}, \chi_{R}, t\right)}$,

$$
e^{S\left(\chi_{L}, \chi_{R}, t\right)} \equiv \int_{-\infty}^{\infty} \mathcal{P}_{t}\left(\omega_{L}, \omega_{R}\right) e^{i \omega_{L} \chi_{L}} e^{i \omega_{R} \chi_{R}} d \omega_{L} d \omega_{R}
$$

This expression generalizes Eq. (22) to the non-markovian case. It can be formally expressed by an inverse Laplace transform of Eq. (45) [28],

$$
\left.e^{S\left(\chi_{L}, \chi_{R}, t\right)}=\frac{1}{2 \pi i} \int_{a-i \infty}^{a+i \infty} d z e^{z t}\langle I| \frac{1}{z-\hat{W}\left(\chi_{L}, \chi_{R}, z\right)}\left(z Z\left(\chi_{L}, \chi_{R}, z\right)\right\rangle\right)_{z \rightarrow \infty} .
$$

Here $a$ is a real number, larger than the real part of all the singularities of the integrand. Equation (51) is a formal result. In practice, it is evaluated as follows: First, we note that the stationary solution (assumed to be unique) of Eq. (44) is given by the eigenvector corresponding to the zero eigenvalue of $\hat{W}$,

$$
\hat{W}\left(\chi_{L}=0, \chi_{R}=0, z=0\right)\left|Z_{S S}\right\rangle=0
$$

Furthermore, as a result of the normalization and conservation of the total spin probabilities, an eigenvalue of $\hat{W}$ satisfies $\lambda_{0}\left(\chi_{L}=0, \chi_{R}=0, z\right)=0$, for all $z$ [28]. This can be directly verified in our case, Eq. (48): The element $\alpha^{+}\left(\alpha^{-}\right)$reduces to $\gamma^{+}\left(\gamma^{-}\right)$when $\chi_{L, R}=0$, and a zero eigenvalue sustains, irrespective of the value of $z$. At finite value for the counting fields an eigenvalue $\lambda_{0}\left(\chi_{L}, \chi_{R}, z\right)$ adiabatically develops from this zero eigenvalue, with small $\chi_{L}, \chi_{R}$ and $z$. The long time behavior of the characteristic function is therefore determined by the pole structure of $\left(z-\lambda_{0}\left(\chi_{1}, \chi_{2}, z\right)\right)^{-1}$ close to zero. This pole $z_{0}\left(\chi_{L}, \chi_{R}\right)$ solves

$$
z_{0}=\lambda_{0}\left(\chi_{L}, \chi_{R}, z_{0}\right)
$$


and it should reduce to $z_{0}\left(\chi_{L}=0, \chi_{R}=0\right)=0$, describing the stationary state. Since all other singularities have larger negative real parts, this pole determines the long time behavior of the characteristic function as

$$
e^{S\left(\chi_{L}, \chi_{R}, t\right)} \rightarrow f\left(\chi_{L}, \chi_{R}, z_{0}\right) e^{z_{0}\left(\chi_{L}, \chi_{R}\right) t}
$$

In the markovian limit $\lambda_{0}$ does not depend on the $z$ variable, thus trivially $z_{0}\left(\chi_{L}, \chi_{R}\right)=$ $\lambda_{0}\left(\chi_{L}, \chi_{R}\right)$.

Concluding, the scheme to obtain the CGF proceeds as follows [28]: (i) We obtain $\lambda_{0}\left(\chi_{L}, \chi_{R}, z\right)$, the eigenvalue of $\hat{W}\left(\chi_{L}, \chi_{R}, z\right)$ that adiabatically develops from the zero (stationary) eigenvalue. (ii) We solve Eq. (53) and obtain the pole $z_{0}\left(\chi_{L}, \chi_{R}\right)$. (iii) We identify the CGF, the analog of Eq. (22), by the pole,

$$
G\left(\chi_{L}, \chi_{R}\right) \equiv z_{0}\left(\chi_{L}, \chi_{R}\right)
$$

Back to (47), we resolve the eigenvalue

$$
\lambda_{0}\left(\chi_{L}, \chi_{R}, z\right)=-\frac{\gamma^{+}+\gamma^{-}}{2}+\frac{\sqrt{\left(\gamma^{+}-\gamma^{-}\right)^{2}+4 \alpha^{+} \alpha^{-}}}{2},
$$

satisfying $\lambda_{0}\left(\chi_{L}=0, \chi_{R}=0, z\right)=0$ for all $z$ [28]. The elements of Eq. (56) all depend on the variable $z, \alpha^{ \pm}$further depend on the counting fields. In principle, we should now solve Eq. (53) in order to gain the CGF, thus the current and its cumulants.

\section{B. Heat Current}

In the long time limit the combination of Eqs. (51) and (54) leads to

$$
z_{0}\left(\chi_{L}, \chi_{R}\right) \rightarrow \frac{1}{t} \ln \int_{-\infty}^{\infty} \mathcal{P}_{t}\left(\omega_{L}, \omega_{R}\right) e^{i \omega_{L} \chi_{L}} e^{i \omega_{R} \chi_{R}} d \omega_{L} d \omega_{R}
$$

It is argued in Ref. [28] that the current and its cumulants can be obtained directly through the analysis of $\lambda_{0}$ itself, Taylor expanded around $z=0, \chi_{L}=0$, and $\chi_{R}=0$,

$$
\lambda_{0}\left(\chi_{L}, \chi_{R}, z\right)=\sum_{n, m, l} \frac{\left(i \chi_{L}\right)^{n}}{n !} \frac{\left(i \chi_{R}\right)^{m}}{m !} \frac{z^{l}}{l !} c^{(n, m, l)}
$$

with

$$
c^{(n, m, l)}=\left.\partial_{\left(i \chi_{L}\right)}^{n} \partial_{\left(i \chi_{R}\right)}^{m} \partial_{z}^{l} \lambda_{0}\left(\chi_{L}, \chi_{R}, z\right)\right|_{\chi_{L}, \chi_{R}, z \rightarrow 0}
$$


The thermal current, calculated by counting energy flow at the $L$ contact, is given by

$$
\left\langle J_{L}\right\rangle \equiv \frac{\left\langle\omega_{L}\right\rangle_{t}}{t}=c^{(1,0,0)} .
$$

Similarly, the current detected at the $R$ contact satisfies

$$
\left\langle J_{R}\right\rangle \equiv \frac{\left\langle\omega_{R}\right\rangle_{t}}{t}=c^{(0,1,0)},
$$

or explicitly

$$
\left\langle J_{R}\right\rangle=\left.\left(\gamma^{+}+\gamma^{-}\right)^{-1}\left[\alpha^{-} \frac{\partial \alpha^{+}}{\partial\left(i \chi_{R}\right)}+\alpha^{+} \frac{\partial \alpha^{-}}{\partial\left(i \chi_{R}\right)}\right]\right|_{\chi_{L}, \chi_{R}, z=0} .
$$

It can be easily verified that this two quantities are identical (with opposite sign), and equivalent to the markovian result (B4). It is thus significant to note that our formalism provides a general expression for the energy current, for many-body systems satisfying the dynamics (4), irrespective of the details of the thermal reservoirs. One can similarly calculate high order cumulants, by evaluating high order $c$ terms [28].

\section{Fluctuation Theorem}

The assumption of no memory enforces a strict energy conservation condition for processes between the system and the environments. In contrast, in the non-markovian regime there is no such an energy-conservation statement, thus it is not obvious that the general FT symmetry (34) still holds for any time interval $t[22,23]$. We now prove that the eigenvalue $\lambda_{0}\left(\chi_{L}, \chi_{R}, z\right)$ satisfies the symmetry relation

$$
\lambda_{0}\left(\chi_{L}, \chi_{R}, z\right)=\lambda_{0}\left(i \beta_{L}-\chi_{L}, i \beta_{R}-\chi_{R}, z\right) .
$$

Only in the markovian limit the symmetry is given in terms of the affinity, as $\lambda_{0}(\chi)=$ $\lambda_{0}(i \Delta \beta-\chi)$. Since the counting fields and $z$ are independent variables, these symmetry relations translate into the analogous relations for the CGF itself, $z_{0}\left(\chi_{L}, \chi_{R}\right)$. This result exemplifies that while microreversibility is sufficient for deriving the basic symmetry relation (63), the SSFT holds only under more restrictive conditions, dictated here by the bath relaxation timescale $[21,23]$.

The symmetry of $\lambda_{0}\left(\chi_{L}, \chi_{R}, z\right)$, thus the symmetry of the CGF, is coded in the product of terms that depend on the counting fields, $\mathcal{D}\left(\chi_{L}, \chi_{R}, z\right) \equiv \alpha^{+} \alpha^{-}$, see Eq. (56). We can 
readily confirm that

$$
\begin{aligned}
& \alpha^{+}\left(i \beta_{L}-\chi_{L}, i \beta_{R}-\chi_{R}, z\right)= \\
& \frac{1}{2 \pi^{2}} \int_{-\infty}^{\infty} \int_{-\infty}^{\infty} e^{-i \omega_{1} \chi_{L}} e^{-\beta_{L} \omega_{1}} e^{-i \omega_{2} \chi_{R}} e^{-\beta_{R} \omega_{2}} C_{R}\left(\omega_{2}\right) C_{L}\left(\omega_{1}\right) \frac{z}{z^{2}+\left(\omega_{0}+\omega_{1}+\omega_{2}\right)^{2}} d \omega_{1} d \omega_{2} \\
& =\alpha^{-}\left(\chi_{L}, \chi_{R}, z\right) .
\end{aligned}
$$

This result is obtained by changing variables, $\omega_{1} \rightarrow-\omega_{1}$ and $\omega_{2} \rightarrow-\omega_{2}$, then utilizing the detailed balance relation, $C_{\nu}(-\omega)=C_{\nu}(\omega) e^{-\beta_{\nu} \omega}$. Similarly, it can be proved that

$$
\alpha^{-}\left(i \beta_{L}-\chi_{L}, i \beta_{R}-\chi_{R}, z\right)=\alpha^{+}\left(\chi_{L}, \chi_{R}, z\right)
$$

As a result, the symmetry relation (63) is confirmed, and the CGF, reached by solving Eq. (56), similarly satisfies

$$
z_{0}\left(\chi_{L}, \chi_{R}\right)=z_{0}\left(i \beta_{L}-\chi_{L}, i \beta_{R}-\chi_{R}\right)
$$

The probability itself is given by an inverse Fourier transform [Eq. (50)] with respect to both fields,

$$
\mathcal{P}_{t}\left(\omega_{L}, \omega_{R}\right)=\frac{1}{(2 \pi)^{2}} \int_{-\infty}^{\infty} \int_{-\infty}^{\infty} e^{z_{0}\left(\chi_{L}, \chi_{R}\right) t} e^{-i \omega_{L} \chi_{L}} e^{-i \omega_{R} \chi_{R}} d \chi_{L} d \chi_{R}
$$

Based on the symmetry of the CGF, it can be readily proved that in the long time limit the following "basic" fluctuation relation holds [22]

$$
\frac{\mathcal{P}_{t}\left(\omega_{L}, \omega_{R}\right)}{\mathcal{P}_{t}\left(-\omega_{L},-\omega_{R}\right)}=e^{\beta_{L} \omega_{L}} e^{\beta_{R} \omega_{R}}
$$

The "standard" fluctuation theorem, expressed in terms of the affinity $\Delta \beta=\beta_{R}-\beta_{L}$ is regained when the kernel $\hat{W}$ reduces to the markovian result; The two counting fields then trivially count the same amount of energy, $\omega_{L}=-\omega_{R}$. This can be explicitly shown by evaluating the elements $\alpha^{+}$and $\alpha^{-}$in the markovian limit $z=0$. We find that

$$
\begin{aligned}
& \alpha^{+}\left(\chi_{L}, \chi_{R}, z=0\right)=\frac{1}{2 \pi} \int_{-\infty}^{\infty} e^{-i\left(\omega_{0}+\omega_{2}\right) \chi_{L}} e^{i \omega_{2} \chi_{R}} C_{R}\left(\omega_{2}\right) C_{L}\left(-\omega_{0}-\omega_{2}\right) d \omega_{2} \\
& \alpha^{-}\left(\chi_{L}, \chi_{R}, z=0\right)=\frac{1}{2 \pi} \int_{-\infty}^{\infty} e^{i\left(\omega_{0}-\omega_{2}\right) \chi_{L}} e^{i \omega_{2} \chi_{R}} C_{R}\left(\omega_{2}\right) C_{L}\left(\omega_{0}-\omega_{2}\right) d \omega_{2}
\end{aligned}
$$

We now define a new counting field, $\chi=\chi_{R}-\chi_{L}$, and immediately verify that the product of these two objects, $\mathcal{D}$, satisfies

$$
\mathcal{D}(\chi)=\mathcal{D}(i \Delta \beta-\chi)
$$


This directly implies on the same symmetry for the markovian CGF,

$$
\lambda_{0}\left(\chi_{R}-\chi_{L}\right)=\lambda_{0}\left(i \Delta \beta-\chi_{R}+\chi_{L}\right),
$$

leading to the standard heat exchange SSFT, Eq. (36).

One should note that Eq. (54) has already involved the assumption of long times, such that only one eigenvalue of $\hat{W}$, with the smallest (absolute) real value, dictates the dynamics. The $z$ dependence in Eq. (48) thus manifests itself when the bath decorrelation time is long, comparable with the inverse relaxation rates of the subsystem. This observation establishes the regime of validity of the SSFT, Eq. (34). It holds when the interval $t$ is long, beyond the bath memory time. We recall that for strictly harmonic systems one directly obtains the SSFT [27], without any reference to the bath characteristic timescale. This is because in coherent systems the reservoirs only serve as a source for excitations, which then elastically cross the impurity. In contrast, in the present model inelastic bath-induced processes are involved in the energy transfer process, making the bath decorrelation time a relevant parameter for the dynamics.

\section{CONCLUSIONS}

We presented here a scheme for obtaining the CGF, thus the current and its moments for the non-equilibrium spin-boson model, an eminent many-body impurity model. A heat exchange SSFT was established for quantum systems incorporating strong system-bath interactions and anharmonic effects. Our derivation relays on the NIBA equations, originally developed for the equilibrium spin-boson model, generalized to describe the dynamics of a spin impurity coupled to multiple thermal reservoirs. Our study provides closed expressions for the CGF, useful for deriving the distribution of heat fluctuations, the averaged current and the thermal noise power. We also showed explicitly that the timescale controlling the onset of the SSFT is the decorrelation time of the reservoirs. Future work will be devoted to generalizing our study to systems showing coherence effects, either using path integral formulation, or quantum master equation methods. Exploring the analogous dynamics for a fermionic system under voltage and temperature biases will be the topic of future studies. 


\section{Acknowledgments}

The research of YN was funded by the Early Research Award of DS. DS further acknowledges support from NSERC discovery grant.

\section{Appendix A: Derivation of EOM for the resolved probability}

The equation of motion for the resolved probabilities in the markovian limit, Eq. (16), are explained here, based on the population dynamics (9). For clarity, we include this equation again,

$$
\dot{p}_{1}(t)=-p_{1}(t) C\left(\omega_{0}\right)+p_{0}(t) C\left(-\omega_{0}\right) .
$$

The population of each state at time $t$ can be expressed in terms of the resolved probability $\mathcal{P}_{t}(n, \omega), n=0,1$, that within the time $t$ a total of energy $\omega$ has been transferred from the left bath to the right bath, while the spin is populating the $n(n=0,1)$ state at time $t$,

$$
\begin{aligned}
& p_{1}(t)=\int_{-\infty}^{\infty} \mathcal{P}_{t}(1, \omega) d \omega, \\
& p_{0}(t)=\int_{-\infty}^{\infty} \mathcal{P}_{t}(0, \omega) d \omega .
\end{aligned}
$$

Plugging these integrals in the dynamical equation (A1), it becomes (ignoring $1 / 2 \pi$ factors for simplicity)

$$
\begin{aligned}
\frac{d}{d t} \int_{-\infty}^{\infty} \mathcal{P}_{t}(1, \omega) d \omega & =-\int_{-\infty}^{\infty} C_{L}\left(\omega_{0}-\omega_{1}\right) C_{R}\left(\omega_{1}\right) d \omega_{1} \times \int_{-\infty}^{\infty} \mathcal{P}_{t}\left(1, \omega_{2}\right) d \omega_{2} \\
& +\int_{-\infty}^{\infty} C_{L}\left(-\omega_{0}-\omega_{1}\right) C_{R}\left(\omega_{1}\right) d \omega_{1} \times \int_{-\infty}^{\infty} \mathcal{P}_{t}\left(0, \omega_{2}\right) d \omega_{2} .
\end{aligned}
$$

We now equate identical energy terms, thus get the resolved dynamics (16)

$$
\begin{aligned}
\frac{d \mathcal{P}_{t}(1, \omega)}{d t} & =-\mathcal{P}_{t}(1, \omega) \int_{-\infty}^{\infty} C_{L}\left(\omega_{0}-\omega_{1}\right) C_{R}\left(\omega_{1}\right) d \omega_{1} \\
& +\int_{-\infty}^{\infty} \mathcal{P}_{t}\left(0, \omega_{1}\right) C_{L}\left(-\omega_{0}-\omega+\omega_{1}\right) C_{R}\left(\omega-\omega_{1}\right) d \omega_{1} .
\end{aligned}
$$

For further validating this equation, we attempt to recover Eq. (A1) by integrating this equation over frequency. The first term in Eq. (A4) trivially reduces to the first term in (A3). The second term in Eq. (A3) is restored following a variable change,

$$
\begin{aligned}
& \int_{-\infty}^{\infty} d \omega \int_{-\infty}^{\infty} \mathcal{P}_{t}\left(0, \omega_{1}\right) C_{L}\left(-\omega_{0}-\omega+\omega_{1}\right) C_{R}\left(\omega-\omega_{1}\right) d \omega_{1}= \\
& \int_{-\infty}^{\infty} d \omega_{2} C_{L}\left(-\omega_{0}-\omega_{2}\right) C_{R}\left(\omega_{2}\right) \int_{-\infty}^{\infty} d \omega \mathcal{P}_{t}\left(0, \omega-\omega_{2}\right)=C\left(-\omega_{0}\right) p_{0}(t)
\end{aligned}
$$




\section{Appendix B: Derivation of the current and its noise power in the markovian limit}

We derive here the steady-state heat current, Eq. (26), and the noise power Eq. (28). We begin by solving the kinetic equations (9) in the long-time limit. The steady-state populations are given by

$$
\begin{aligned}
& p_{1}=\frac{k_{u}}{k_{u}+k_{d}}=\frac{C\left(-\omega_{0}\right)}{C\left(-\omega_{0}\right)+C\left(\omega_{0}\right)} \\
& p_{0}=\frac{k_{d}}{k_{u}+k_{d}}=\frac{C\left(\omega_{0}\right)}{C\left(-\omega_{0}\right)+C\left(\omega_{0}\right)} .
\end{aligned}
$$

We now study the first derivative of the CGF, Eq. (24), with respect to the counting field,

$$
\begin{aligned}
\langle J\rangle=\left.\frac{d G(\chi)}{d(i \chi)}\right|_{\chi=0} & =\left[\frac{d C^{d}(\chi)}{d i \chi} C^{u}(\chi)+\frac{d C^{u}(\chi)}{d i \chi} C^{d}(\chi)\right] \\
& \times\left.\left[\left(C\left(\omega_{0}\right)-C\left(\omega_{0}\right)\right)^{2}+4 C^{d}(\chi) C^{u}(\chi)\right]^{-1 / 2}\right|_{\chi=0} .
\end{aligned}
$$

Note that $C^{d}(\chi=0)=C\left(\omega_{0}\right)$ and $C^{u}(\chi=0)=C\left(-\omega_{0}\right)$, a direct result of Eqs. (20) and (21). We identify the second term in the expression above by the sum $\left(C\left(\omega_{0}\right)+C\left(-\omega_{0}\right)\right)^{-1}$. The partial derivatives are given by (ignoring $(2 \pi)^{-1}$ factors for simplicity)

$$
\begin{aligned}
& \left.\frac{d C^{u}(\chi)}{d(i \chi)}\right|_{\chi=0}=\int_{-\infty}^{\infty} \omega C_{R}(\omega) C_{L}\left(-\omega-\omega_{0}\right) d \omega=-\int_{-\infty}^{\infty} \omega C_{R}(-\omega) C_{L}\left(\omega-\omega_{0}\right) d \omega \\
& \left.\frac{d C^{d}(\chi)}{d(i \chi)}\right|_{\chi=0}=\int_{-\infty}^{\infty} \omega C_{R}(\omega) C_{L}\left(\omega_{0}-\omega\right) d \omega .
\end{aligned}
$$

Plugging these terms into Eq. (B2) we find the current

$$
\begin{aligned}
\langle J\rangle & =\frac{1}{C\left(\omega_{0}\right)+C\left(-\omega_{0}\right)}\left[C\left(-\omega_{0}\right) \int_{-\infty}^{\infty} \omega C_{R}(\omega) C_{L}\left(\omega_{0}-\omega\right) d \omega\right. \\
& \left.-C\left(\omega_{0}\right) \int_{-\infty}^{\infty} \omega C_{R}(-\omega) C_{L}\left(\omega-\omega_{0}\right) d \omega\right] .
\end{aligned}
$$

It is significant to note that this expression stays intact for non-markovian systems [28]. Next we adopt the steady-state population (B1) and simplify the result,

$$
\langle J\rangle=p_{1} \int_{-\infty}^{\infty} \omega C_{R}(\omega) C_{L}\left(\omega_{0}-\omega\right) d \omega-p_{0} \int_{-\infty}^{\infty} \omega C_{R}(-\omega) C_{L}\left(\omega-\omega_{0}\right) d \omega,
$$

which is precisely Eq. (26). We now verify that $\langle J(\Delta T)\rangle=-\langle J(-\Delta T)\rangle$ for a spatially symmetric system. Upon exchange of the temperature polarity, the above expression becomes

$$
\langle J(-\Delta T)\rangle=p_{1} \int_{-\infty}^{\infty} \omega C_{L}(\omega) C_{R}\left(\omega_{0}-\omega\right) d \omega-p_{0} \int_{-\infty}^{\infty} \omega C_{L}(-\omega) C_{R}\left(\omega-\omega_{0}\right) d \omega .
$$


We change variables, $\omega-\omega_{0}=-x$, and get

$$
\langle J(-\Delta T)\rangle=p_{1} \int_{-\infty}^{\infty}\left(\omega_{0}-x\right) C_{L}\left(\omega_{0}-x\right) C_{R}(x) d x-p_{0} \int_{-\infty}^{\infty}\left(\omega_{0}-x\right) C_{L}\left(x-\omega_{0}\right) C_{R}(-x) d x
$$

This expression can be organized as

$$
\begin{aligned}
\langle J(-\Delta T)\rangle & =\omega_{0}\left[p_{1} \int_{-\infty}^{\infty} C_{L}\left(\omega_{0}-x\right) C_{R}(x) d x-p_{0} \int_{-\infty}^{\infty} C_{L}\left(x-\omega_{0}\right) C_{R}(-x) d x\right] \\
& -\left[p_{1} \int_{-\infty}^{\infty} x C_{L}\left(\omega_{0}-x\right) C_{R}(x) d x-p_{0} \int_{-\infty}^{\infty} x C_{L}\left(x-\omega_{0}\right) C_{R}(-x) d x\right] .
\end{aligned}
$$

Since the first line fade away once combining the definition (11) and the steady-state population (27), we establish the odd symmetry for the current with $\Delta T$. The noise power is formally given by

$$
\begin{aligned}
& \langle S\rangle=\left.\frac{d^{2} G(\chi)}{d(i \chi)^{2}}\right|_{\chi=0} \\
& =\left[\frac{d^{2} C^{d}(\chi)}{d(i \chi)^{2}} C^{u}(\chi)+\frac{d^{2} C^{u}(\chi)}{d(i \chi)^{2}} C^{d}(\chi)+2 \frac{d C^{u}(\chi)}{d i \chi} \frac{d C^{d}(\chi)}{d i \chi}\right] \\
& \times\left.\left[\left(C\left(\omega_{0}\right)-C\left(-\omega_{0}\right)\right)^{2}+4 C^{d}(\chi) C^{u}(\chi)\right]^{-1 / 2}\right|_{\chi=0} \\
& -\left.2\left[\frac{d C^{d}(\chi)}{d(i \chi)} C^{u}(\chi)+\frac{d C^{u}(\chi)}{d(i \chi)} C^{d}(\chi)\right]^{2}\left[\left(C\left(\omega_{0}\right)-C\left(-\omega_{0}\right)\right)^{2}+4 C^{d}(\chi) C^{u}(\chi)\right]^{-3 / 2}\right|_{\chi=0}
\end{aligned}
$$

Using the explicit expressions for the correlations we reduce it to

$$
\begin{aligned}
\langle S\rangle= & p_{1} \int_{-\infty}^{\infty} \omega^{2} C_{R}(\omega) C_{L}\left(\omega_{0}-\omega\right) d \omega+p_{0} \int_{-\infty}^{\infty} \omega^{2} C_{R}(-\omega) C_{L}\left(\omega-\omega_{0}\right) d \omega+ \\
& -2 \frac{1}{C\left(\omega_{0}\right)+C\left(-\omega_{0}\right)} \int_{-\infty}^{\infty} \omega C_{R}(-\omega) C_{L}\left(\omega-\omega_{0}\right) d \omega \int_{-\infty}^{\infty} \omega C_{R}(\omega) C_{L}\left(\omega_{0}-\omega\right) d \omega+ \\
& -2 \frac{1}{C\left(\omega_{0}\right)+C\left(-\omega_{0}\right)}\langle J\rangle^{2} .
\end{aligned}
$$

\section{Appendix C: The spin-boson model in the weak coupling limit}

We study here the counting statistics of the unbiased $\left(\omega_{0}=0\right)$ spin-boson model, and verify the validity of the SSFT in this case, both under the Born-Markov Approximation $[25,26]$. Our starting point is the SB Hamiltonian [Eq. (2)]. We take $\omega_{0}=0$ and apply a 
unitary transformation

$$
U^{\dagger} \sigma_{z} U=\sigma_{x}, \quad U^{\dagger} \sigma_{x} U=\sigma_{z}
$$

with $U=\frac{1}{\sqrt{2}}\left(\sigma_{x}+\sigma_{z}\right)$, to obtain the transformed Hamiltonian $H_{W}=U^{\dagger} H U$,

$$
\begin{aligned}
H_{W} & =H_{0}+H_{I}+H_{B} \\
H_{0} & =\frac{\Delta}{2} \sigma_{z} ; \quad H_{I}=\sigma_{x} \sum_{\nu, j} \lambda_{j, \nu}\left(b_{j, \nu}^{\dagger}+b_{j, \nu}\right) \\
H_{B} & =\sum_{\nu} H_{\nu} ; \quad H_{\nu}=\sum_{j} \omega_{j} b_{j, \nu}^{\dagger} b_{j, \nu} .
\end{aligned}
$$

Note that the subsystem energy gap is now given by $\Delta$, rather than $\omega_{0}$ as in the original spin-boson description [Eq. (2)]. This form is a convenient starting point for a perturbationtheory calculation, assuming weak system-bath coupling. We outline next the principles of this standard approach [1]. Details, for the two-bath scenario, can be found in Ref. [13]. We begin with an equation of motion for the total density matrix $\rho(t)$ in the interaction representation,

$$
\dot{\rho}=-i\left[H_{I}(t), \rho(t)\right]
$$

The operators are given by $O(t)=e^{i\left(H_{0}+H_{B}\right) t} O e^{-i\left(H_{0}+H_{B}\right) t}$. We now make the following assumptions: (i) At the initial time the reservoirs are (separately) maintained in thermal equilibrium, isolated from the subsystem, and (ii) the spin and the baths are weakly coupled, allowing for a weak-coupling expansion with respect to the interaction term in Eq. (C2). This results in

$$
\dot{\rho}_{S}(t)=-\int_{0}^{t} d s \operatorname{Tr}\left[H_{I}(t),\left[H_{I}(s), \rho_{S}(s) \otimes \rho_{B}\right]\right]
$$

which is a non-markovian equation of motion. Here $\rho_{S}(t)=\operatorname{Tr}[\rho(t)]$ is the reduced density matrix of the system; the trace is performed over the two reservoirs. The bath density matrix is a product state, $\rho_{B}=\rho_{L} \otimes \rho_{R}$, of the two canonical density matrices $\rho_{\nu}=$ $e^{-H_{\nu} / T_{\nu}} / \operatorname{Tr}_{\nu}\left[e^{-H_{\nu} / T_{\nu}}\right]$. In our model $(\mathrm{C} 2)$ the population dynamics becomes decoupled from the coherences dynamics. It obeys

$$
\dot{p}_{1}=-2 \Re \int_{0}^{t} e^{i \Delta(t-s)} g(t-s) p_{1}(s) d s+2 \Re \int_{0}^{t} e^{-i \Delta(t-s)} g(t-s) p_{0}(s) d s .
$$


Here $p_{n}(t)=\left(\rho_{S}(t)\right)_{n, n}(n=0,1)$ and $g(\tau)=\sum_{\nu} g_{\nu}(\tau)$, with

$$
g_{\nu}(\tau)=\int_{0}^{\infty} \frac{J_{\nu}(\omega)}{4 \pi}\left[n_{\nu}(\omega) e^{i \omega \tau}+\left(n_{\nu}(\omega)+1\right) e^{-i \omega \tau}\right] d \omega .
$$

The bath spectral function is given by $J_{\nu}(\omega)=4 \pi \sum_{j} \lambda_{j, \nu}^{2} \delta\left(\omega-\omega_{j}\right)$ and the function $n_{\nu}(\omega)=\left[e^{\beta_{\nu} \omega}-1\right]^{-1}$ denotes the Bose-Einstein distribution. We now make the markovian approximation, assuming that bath correlations decay on a time scale shorter than the subsystem characteristic timescale. This converts Eq. (C5) into a kinetic-Master equation,

$$
\begin{aligned}
& \dot{p}_{1}=-p_{1} \sum_{\nu} k_{1 \rightarrow 0}^{\nu}+p_{0} \sum_{\nu} k_{0 \rightarrow 1}^{\nu} \\
& p_{1}(t)+p_{0}(t)=1
\end{aligned}
$$

where the Fermi-golden rule transition rates are evaluated at the subsystem energy gap $\Delta$, satisfying

$$
k_{0 \rightarrow 1}^{\nu}=\Gamma_{\nu}(\Delta) n_{\nu}(\Delta), \quad k_{1 \rightarrow 0}^{\nu}=\Gamma_{\nu}(\Delta)\left[1+n_{\nu}(\Delta)\right]
$$

The rate $\Gamma_{\nu}(\omega)=2 \pi \sum_{j} \lambda_{j, \nu}^{2} \delta\left(\omega_{j}-\omega\right)$ denotes the temperature independent part of the relaxation rate. The dynamics $(\mathrm{C} 7)$ describes spin flip processes accompanied by an energy transfer at the amount of $\Delta$ to either the left or the right reservoirs.

We proceed and derive the cumulant generating function in the present weak coupling limit following [26]. We begin by defining $\mathcal{P}_{t}(n, q \Delta)$ as the probability that within the time $t$ a total energy $q \Delta$ has been transferred from the left bath to the right bath, while the spin is populating the $n(n=0,1)$ state at time $t$. Note that $q$ here is an integer, since energy is transferred here in discrete quanta of $\Delta$, between the two baths. In other words, whenever the spin flips, the spin gap $\Delta$ is dissipated or absorbed at either the left or the right reservoir. Thus,

$$
\begin{aligned}
& \frac{d \mathcal{P}_{t}(0, q \Delta)}{d t}=-\mathcal{P}_{t}(0, q \Delta)\left(k_{0 \rightarrow 1}^{L}+k_{0 \rightarrow 1}^{R}\right)+\mathcal{P}_{t}(1,(q-1) \Delta) k_{1 \rightarrow 0}^{R}+\mathcal{P}_{t}(1, q \Delta) k_{1 \rightarrow 0}^{L} \\
& \frac{d \mathcal{P}_{t}(1, q \Delta)}{d t}=-\mathcal{P}_{t}(1, q \Delta)\left(k_{1 \rightarrow 0}^{L}+k_{1 \rightarrow 0}^{R}\right)+\mathcal{P}_{t}(0,(q+1) \Delta) k_{0 \rightarrow 1}^{R}+\mathcal{P}_{t}(0, q \Delta) k_{0 \rightarrow 1}^{L}
\end{aligned}
$$

We Fourier transform these equations with the counting field $\chi$ to obtain the characteristic function,

$$
|Z(\chi, t)\rangle \equiv\left(\begin{array}{c}
\sum_{q} \mathcal{P}_{t}(0, q \Delta) e^{i q \Delta \chi} \\
\sum_{q} \mathcal{P}_{t}(1, q \Delta) e^{i q \Delta \chi}
\end{array}\right)
$$


satisfying a first order differential equation,

$$
\frac{d|Z(\chi, t)\rangle}{d t}=-\hat{W}(\chi)|Z(\chi, t)\rangle
$$

with the matrix

$$
\hat{W}=\left(\begin{array}{cc}
k_{0 \rightarrow 1}^{L}+k_{0 \rightarrow 1}^{R} & -k_{1 \rightarrow 0}^{L}-k_{1 \rightarrow 0}^{R} e^{i \chi \Delta} \\
-k_{0 \rightarrow 1}^{L}-k_{0 \rightarrow 1}^{R} e^{-i \chi \Delta} & k_{1 \rightarrow 0}^{L}+k_{1 \rightarrow 0}^{R}
\end{array}\right)
$$

The CGF is given by the negative of the smallest eigenvalues of this matrix,

$$
G(\chi)=\frac{-A+\sqrt{A^{2}+4 B(\chi)}}{2} .
$$

The coefficients are defined as

$$
\begin{aligned}
A & =\Gamma_{L}\left[1+2 n_{L}(\Delta)\right]+\Gamma_{R}\left[1+2 n_{R}(\Delta)\right] \\
B(\chi) & =\Gamma_{L} \Gamma_{R} n_{L}(\Delta) n_{R}(\Delta)\left[\left(e^{-i \chi \Delta}-1\right) e^{\beta_{L} \Delta}+\left(e^{i \chi \Delta}-1\right) e^{\beta_{R} \Delta}\right] .
\end{aligned}
$$

For brevity, we have discarded the direct dependence of the rates on frequency, $\Gamma(\Delta)$. It can be easily verified that the cumulant generating function satisfies the symmetry $G(\chi)=$ $G(i \Delta \beta-\chi)$ with $\Delta \beta=\beta_{R}-\beta_{L}$. This symmetry can be translated into the fluctuation relation at long time $t[21,26]$,

$$
\frac{\mathcal{P}_{t}(q \Delta)}{\mathcal{P}_{t}(-q \Delta)}=e^{q \Delta\left(\beta_{R}-\beta_{L}\right)}
$$

Comparing this result to the strong coupling expression (36), we note that in the unbiasedweak coupling limit the discrete energy $q \Delta$ replaces the continuous variable $\omega$, since the reservoirs here accept or contribute energy in quanta of the spin spacing $\Delta$. Finally, the current and the noise power are given by

$$
\begin{aligned}
& \langle J\rangle=\left.\frac{1}{A} \frac{\partial B}{\partial i \chi}\right|_{\chi=0} \\
& \langle S\rangle=\left.\frac{1}{A}\left[\frac{\partial^{2} B}{\partial(i \chi)^{2}}-\frac{2}{A^{2}}\left(\frac{\partial B}{\partial i \chi}\right)^{2}\right]\right|_{\chi=0} .
\end{aligned}
$$

The elements in this expression are

$$
\begin{aligned}
& \left.\frac{\partial B}{\partial(i \chi)}\right|_{\chi=0}=\Delta \Gamma_{L} \Gamma_{R}\left[n_{L}(\Delta)-n_{R}(\Delta)\right] \\
& \left.\frac{\partial^{2} B}{\partial(i \chi)^{2}}\right|_{\chi=0}=-\Delta^{2} \Gamma_{L} \Gamma_{R}\left[n_{L}(-\Delta) n_{R}(\Delta)+n_{R}(-\Delta) n_{L}(\Delta)\right]
\end{aligned}
$$


We find that in this weak coupling limit the current satisfies

$$
\langle J\rangle=\Delta \frac{\Gamma_{L} \Gamma_{R}\left[n_{L}(\Delta)-n_{R}(\Delta)\right]}{\Gamma_{L}\left[1+2 n_{L}(\Delta)\right]+\Gamma_{R}\left[1+2 n_{R}(\Delta)\right]} .
$$

This result agrees with previous studies [12].

[1] U. Weiss, Quantum Dissipative Systems (World Scientific, Singapore, 1993).

[2] A. J. Legget et al., Rev. Mod. Phys. 59, 1 (1987).

[3] R. A. Marcus, J. Chem. Phys. 24, 966 (1956).

[4] P. Nalbach, A. Ishizaki, G. R. Fleming, and M. Thorwart, New J. Phys. 13, 063040 (2011).

[5] A. C. Hewson, The Kondo Problem to Heavy Fermions, (Cambridge University Press, Cambridge, England, 1993).

[6] A. Mitra and A. J. Millis, Phys. Rev. B 72, 121102(R) (2005).

[7] D. Segal, D. R. Reichman, and A. J. Millis, Phys. Rev. B 76, 195316 (2007).

[8] R. M. Lutcgyn, L. Cyqinski, C. P. Nave, and S. Das Sarma, Phys. Rev. B 78, 024508 (2008).

[9] M. Galperin, M. A. Ratner, A. Nitzan, and A. Troisi, Science 319, 1056 (2008).

[10] N. Makri and D. E. Makarov, J. Chem. Phys. 102, 4600 (1995); N. Makri and D. E. Makarov, J. Chem. Phys. 102, 4611 (1995).

[11] D. Segal, A. J. Millis, and D. R. Reichman, Phys. Rev. B 82, 205323 (2010).

[12] D. Segal and A. Nitzan, Phys. Rev. Lett. 94, 034301 (2005).

[13] D. Segal, Phys. Rev. B 73, 205415 (2006).

[14] T. Ruokola and T. Ojanen, Phys. Rev. B 83, 045417 (2011).

[15] K. A. Velizhanin, M. Thoss, and H. Wang, J. Chem. Phys. 133, 084503 (2010).

[16] K. A. Velizhanin, H. Wang, and M. Thoss, Chem. Phys. Lett. 460, 325 (2008).

[17] C. Jarzynski and D. K. Wöjcik, Phys. Rev. Lett. 92, 230602 (2004).

[18] D. J. Evans and E. G. D. Cohen, and G. P. Morriss, Phys. Rev. Lett. 71, 2401 (1993).

[19] G. Gallavotti and E. G. D. Cohen, Phys. Rev. Lett. 74, 2694 (1995).

[20] Y. Sughiyama and S. Abe, J. Stat Mech. (2008) P05008.

[21] M. Esposito, U. Harbola, and S. Mukamel, Rev. Mod. Phys. 81, 1665 (2009).

[22] M. Campisi, P. Hänggi, and P. Talkner, Rev. Mod. Phys. 83, 771 (2011).

[23] D. Andrieux, P. Gaspard, T. Monnai, and S. Tasaki, New J. Phys. 11, 043014 (2009). 
[24] M. Campisi, P. Hänggi, and P. Talkner, Phys. Rev. Lett. 105, 140601 (2010).

[25] U. Harbola, M. Esposito, and S. Mukamel, Phys. Rev. B 76, 085408 (2007).

[26] J. Ren, P. Hänggi, and B. Li, Phys. Rev. Lett. 104, 170601 (2010).

[27] K. Saito and A. Dhar, Phys. Rev. Lett. 99, 180601 (2007).

[28] C. Flindt, T. Novotny, A. Braggio, M. Sassetti, and A.-P. Jauho, Phys. Rev. Lett. 100, 150601 (2008); C. Flindt, T. Novotny, A. Braggio, and A.-P. Jauho, Phys. Rev. B 82, 155407 (2010).

[29] D. Marcos, C. Emary, T. Brandes, and R. Aguado, Phys. Rev. B 83, 125426 (2011).

[30] C. Emary and R. Aguado, arXiv:1106.5219

[31] T. Kawamoto and N. Hatano, arXiv:1105.3579

[32] H. Dekker, Phys. Rev. A 35, 1436 (1987).

[33] C. Aslangul, N. Pottier, and D. Saint-James, Journal De Physique 47, 757 (1986).

[34] G. D. Mahan, Many-particle physics (Plenum press, New York, 2000).

[35] L. Nicolin and D. Segal, arXiv:1106.0353. 